\title{
An evaluation of early medication use for COPD: a population-based cohort study
}

\author{
This article was published in the following Dove Press journal: \\ International Journal of COPD \\ 7 December 2016 \\ Number of times this article has been viewed
}

Jamie Falk'

Natalia Dik ${ }^{2}$

Shawn Bugden'

'College of Pharmacy, Rady Faculty of Health Sciences, ${ }^{2}$ Manitoba Centre for Health Policy, Department of Community Health Sciences, University of Manitoba, Winnipeg, MB, Canada
Correspondence: Jamie Falk College of Pharmacy, Rady Faculty of Health Sciences, Apotex Centre, University of Manitoba, 750 McDermot Avenue, Winnipeg, MB R3E 0T5, Canada $\mathrm{Tel}+\mathrm{I} 2043185280$

Fax +I 2047893744

Email jamison.falk@umanitoba.ca
Purpose: The aim of this study was to evaluate the first initiation, sequence of addition, and appropriate prescribing of COPD medications in Manitoba, Canada.

Patients and methods: A population-based cohort study of COPD medication use was conducted using administrative health care data (1997-2012). Those aged $\geq 35$ years with COPD based on three or more COPD-related outpatient visits over a rolling 24-month window or at least one COPD-related hospitalization were included. The first medication(s) dispensed on or after the date of COPD diagnosis were determined based on pharmacy claims. The next medication(s) in sequence were determined to be additions or switches to the previous regimen. Evaluation of guideline-based appropriateness to receive inhaled corticosteroids (ICS) was based on exacerbation history and past medication use.

Results: Of 13,369 patients dispensed COPD medications after diagnosis, 66.0\% were dispensed short-acting bronchodilators as first medications. Although long-acting bronchodilators alone were uncommonly used as first or subsequent medications, ICS were dispensed as first medications in $28.2 \%$ of patients. Over the study period, use of short-acting bronchodilators as first medications declined from $70.6 \%$ to $59.4 \%(P<0.0001)$, whereas the use of ICS as a first medication increased from $23.5 \%$ to $34.4 \%(P<0.0001)$. Dispensation of an ICS plus a longacting $\beta$-agonist increased dramatically from $1.2 \%$ to $27.3 \%(P<0.0001)$. By the end of the study period, the majority of patients $(53.3 \%)$ were being initiated on two or more medications. Of 5,823 patients dispensed an ICS, 52.4\% met Canadian guideline criteria for initiating an ICS, whereas $0.3 \%$ met Global Initiative for Chronic Obstructive Lung Disease guideline criteria.

Conclusion: The use of first-line medications has declined over time, replaced primarily by combination inhalers prescribed early without prior trials of appropriate next step medications. This, along with an increasingly predominant use of multiple first medications, indicates a significant degree of medication burden in this already complex patient population.

Keywords: COPD, medication, inhaled steroids, Canada, guideline, appropriate

\section{Introduction}

COPD is a progressive disease leading to a decline in lung function over time despite the best available care. The presence of multiple comorbidities is common among COPD patients, with the vast majority of patients using multiple classes of chronic non-COPD medications. ${ }^{1-3}$ In addition to significant effects on morbidity, COPD is the third leading cause of death in the world. ${ }^{4}$ Unfortunately, with the exceptions of smoking cessation and oxygen therapy in hypoxic patients, no interventions to date have been able to reduce the likelihood of death in patients with COPD. ${ }^{5}$ Furthermore, based on current clinical trial data, the magnitude of effect of pharmacological treatments for COPD has been shown to be relatively limited. ${ }^{6,7}$ 
In comparison to the use of short-acting bronchodilators (SABD) or placebo, the use of single long-acting bronchodilators (LABD) (ie, long-acting $\beta$-agonists [LABA] or longacting muscarinic antagonists [LAMA]) has resulted in small reductions in the rates of exacerbations and hospitalizations without significant adverse events. ${ }^{8,9}$ However, despite the entry of many new agents into the market, numerous clinical trials demonstrate that combining two LABDs offers only modest improvements in patient-relevant outcomes compared to the individual inhalers alone. ${ }^{6,10-13}$ The effectiveness of inhaled corticosteroids (ICS) in the management of COPD remains controversial. Large randomized trials and subsequent meta-analyses have confirmed a modest reduction in acute exacerbations of COPD (AECOPD), but not a reduction in hospitalizations or clinically important improvements in health status with the addition of an ICS to a LABD. Most concerning, however, is a significant increase in the incidence of pneumonias in those using ICS in general and compared to a LABD alone. ${ }^{14-16}$

Based on data from the US from 2010, inhaler prescriptions for COPD patients increased up to three- to fourfold compared to the previous decade. ${ }^{17} \mathrm{~A}$ study of the Veterans Affairs health system from 1999 to 2003 found that of newly diagnosed COPD patients, 29\% were prescribed three to four medication classes over the 1-year follow-up. ${ }^{18}$ A Canadian study of COPD prescribing patterns found that $63 \%$ of patients in Quebec and Ontario with mild COPD had received a prescription for ICS. ${ }^{19}$

These findings lead us to hypothesize that COPD medications of modest efficacy are being initiated and added earlier than may be indicated. To our knowledge, there are no recent large database studies on the first initiation and sequence of addition of COPD medications in the literature.

We conducted a large population-based cohort study of COPD patients in Manitoba, Canada, to characterize prescribing patterns and to evaluate the clinical appropriateness of the initiation and sequencing of COPD medications.

\section{Methods}

The objective of the study was to describe the utilization and sequence of addition of COPD medications in Manitoba between the calendar years 1997 and 2012. A retrospective population-based cohort study of COPD medication use was conducted using administrative health care data from the province of Manitoba, Canada, from April 1, 1997 to March 31, 2013. Data were obtained through the Manitoba Centre for Health Policy, which maintains the province's Population Health Research Data Repository. The repository contains linked administrative health care data for the population of Manitoba (1.3 million, 2013). The databases used included the Drug Program Information Network, a community pharmacy-based prescription processing system that enables submission of online insurance claims by pharmacies, the Medical Services database that contains records of feefor-service medical claims, provincial Hospital Discharge Abstracts containing summary data of each hospital stay, and the Manitoba Health Registry, from which we obtained basic demographics on individuals in our cohort. Scrambled patient identifiers are shared between these databases, enabling linkage of records and allowing for the longitudinal analysis of individual patients across the entire health care system.

The eligible population included Manitobans 35 years of age or older with COPD. To satisfy the definition for COPD, patients had to have three or more COPD-related outpatient visits over a rolling 24-month window or at least one COPDrelated hospitalization with cohort entry from the date of first record. COPD codes were defined using the International Classification of Diseases, Ninth Revision (ICD-9) codes 491, 492, and 496 or 10th Revision (ICD-10) codes J41, $\mathrm{J} 42$, J43, and J44. This case definition has been reported to have a specificity of $95.4 \%$ for identifying patients with COPD. ${ }^{20,21}$ Patients with a diagnosis of asthma (ICD-9 code 493 or ICD-10 code J45) were excluded.

A patient was considered an incident user if he or she received a prescription for a COPD medication after their entry date. A first medication was defined as a prescription dispensed on or after the entry date of the COPD diagnosis. If another medication was dispensed up to 7 days after the first medication, it was also considered to be a part of the first medication. Sequential medications were defined as all prescriptions dispensed 8 or more days since the start of the first medication. To determine whether the second medication dispensed was an addition or a switch, medication dispensations occurring after the second medication were checked. If the second medication was different from the first, but the next medication after the second was the same as the first, then the result was considered an addition. If the second medication was different from the first and the next medication after the second was either different from the first or the first did not occur again (ie, stopped), then the result was considered a switch.

The following medications were considered for our analysis: short-acting $\beta-2$ agonists (SABA), short-acting muscarinic antagonists (SAMA), fixed-dose combination inhalers of SABA and SAMA, LABA, LAMA, methylxanthines, ICS, and fixed-dose combination inhalers of ICS and LABA (Table S1). 
For the evaluation of guideline-based appropriateness to receive an ICS, in order for a patient to meet the criteria recommended by the 2008 Canadian Thoracic Society (CTS) COPD guidelines for ICS initiation, it was necessary to have had at least one AECOPD in the past year or be inadequately controlled on a LABA, LAMA, and an as-needed SABD. ${ }^{22}$ Since it is not possible to determine adequate control using the database, having a dispensing claim for these medications was considered to meet the criteria. An AECOPD was defined as a hospital admission for COPD, or a COPD claim plus a prescription for antibiotics and/or systemic corticosteroids within 7 days of that claim. Antibiotic or systemic corticosteroid prescriptions with a duration beyond 21 days were considered chronic use and excluded. In order for a patient to meet the two criteria recommended by the Global Initiative for Chronic Obstructive Lung Disease (GOLD) COPD guidelines for ICS initiation, it was necessary to have had at least two AECOPD or at least one COPD hospitalization in the past year in addition to having inadequate control of COPD on LABDs. ${ }^{23}$ To define two separate exacerbation episodes, the antibiotic and/or systemic corticosteroid prescriptions were required to be greater than 14 days apart.

$\chi^{2}$ test were used to compare COPD medication use at the beginning and at the end of the study period. SAS ${ }^{\circledR}$ version 9.4 (SAS Institute, Cary, NC, USA) and Microsoft Excel $2013^{\circledR}$ (Microsoft Corporation, Redmond, WA, USA) were used for data analysis. Approvals were obtained from the University of Manitoba Health Research Ethics Board (HREB\# HS18642 [H2015:231]) and the Manitoba Health Information Privacy Committee (HIPC\# 2015/2016-17). These committees do not require individual consent for research conducted using de-identified administrative data when reasonable safeguards to protect confidentiality and security of personal health information are in place.

\section{Results}

A total of 19,367 patients satisfied the case definition of COPD from 1997 to 2012. The median age of the population was 73 years at the time of diagnosis and did not change significantly over the study period. Fifty-seven percent of the patients were male. In total, 13,369 patients (69\%) were dispensed a COPD medication after their diagnosis. Of these patients, for the majority $(n=8,825)$, the first medication dispensed was a SABD (SABA alone, SAMA alone, or combined inhaler of both) (Table 1). LABA and LAMA inhalers alone were uncommonly used as first medications, while ICS were used in more than a quarter of patients. Over the study period, use of a SABD as first medications declined
Table I Classes of first medications after diagnosis over study period

\begin{tabular}{ll}
\hline Medication & $\begin{array}{l}\text { \% of users } \\
(\mathbf{n}=1 \mathbf{3 , 3 6 9})^{\mathbf{a}}\end{array}$ \\
\hline SABD & 66.0 \\
SABA alone & 25.5 \\
SAMA alone & 13.4 \\
SABA + SAMA alone & 27.2 \\
LABA ( \pm SABD, without ICS) & 2.3 \\
LAMA ( \pm SABD, without ICS) & $\mathbf{2 . 1}$ \\
ICS (any) & $\mathbf{2 8 . 2}$ \\
ICS + LABA & 14.7 \\
Others (methylxanthines, LABA + LAMA) & 1.5 \\
\hline
\end{tabular}

Note: ${ }^{\mathrm{T}}$ Total of bolded figures $=100 \%$.

Abbreviations: SABD, short-acting bronchodilators; SABA, short-acting $\beta-2$ agonists; SAMA, short-acting muscarinic antagonist; LABA, long-acting $\beta$-2 agonists; LAMA, long-acting muscarinic antagonist; ICS, inhaled corticosteroids.

from $70.6 \%$ in those diagnosed in 1997 to $59.4 \%$ for those diagnosed in $2012(P<0.0001)$. The use of LABA alone remained consistently low throughout the study period, and LAMA use rose slightly from almost no use $(0.1 \%)$ for those diagnosed in 1997 (the first LAMA was marketed in 2002) to $5.5 \%$ for those diagnosed in $2012(P<0.0001)$. However, the use of ICS as a first medication increased significantly from $23.5 \%$ in those diagnosed in 1997 to $34.4 \%$ for those diagnosed in $2012(P<0.0001)$. An increase in the use of an ICS in combination with a LABA was most remarkable, rising from $1.2 \%$ in those diagnosed in 1997 to $27.3 \%$ in those diagnosed in $2012(P<0.0001)$ (Figure 1).

Of those patients who were dispensed medications, $46.9 \%$ were initiated on one medication, $41.0 \%$ were

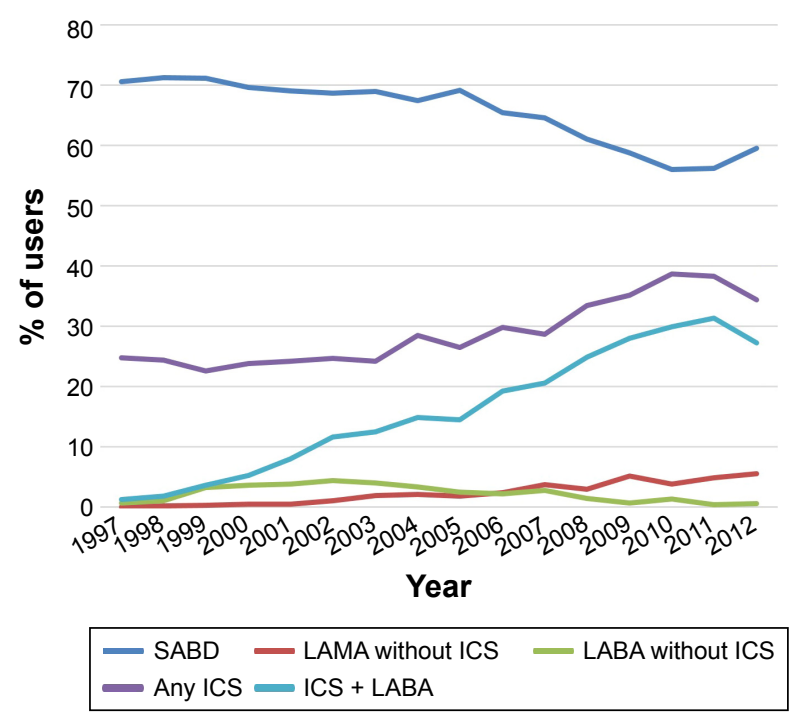

Figure I Classes of first medications after diagnosis by year.

Abbreviations: SABD, short-acting bronchodilator; LAMA, long-acting muscarinic antagonist; LABA, long-acting $\beta$-agonist; ICS, inhaled corticosteroid. 
initiated on two, and $12.1 \%$ were initiated on three or more first medications. Analysis of this population over time revealed that $57 \%$ of patients were initiated on one medication, $36.9 \%$ initiated on two, and $6.1 \%$ initiated on three or more first medications if diagnosed in 1997. For diagnosis in 2012, those initiated on one medication had declined to $46.8 \%$, those initiated on two medications rose modestly to $40.0 \%$, and those initiated on three or more medications more than doubled to $13.3 \%(P<0.0001$ for all three comparisons of 1997-2012) (Figure 2).

Of the 13,369 patients who started medications, 2,013 (15.1\%) stopped any COPD medications that were initially started and did not have any further medications initiated throughout their time in the study. Of those who stopped medications, most were SABD users $(1,482)$, contributing to a decline in the number of SABD users from 8,825 for first medication users to 7,280 SABD users after the first series of medication changes took place. Among the initial SABD users, 1,143 changes were made. Of those changes, $952(83.3 \%)$ involved a switch to or addition of an ICS. Of these changes involving an ICS, 499 (52.4\%) involved a switch to or addition of a LABA + ICS combination. Use of ICS overall decreased modestly from 3,764 patients $(28.2 \%)$ to 3,329 patients $(24.9 \%)(P<0.0001)$, while use of the ICS + LABA combination remained relatively stable with 1,966 initial users of this combination and 1,848 users after the first series of medication changes took place $(P<0.0001)$. Again, LABA and LAMA use in the absence of ICS was minimal, accounting for $244(1.8 \%)$ and $341(2.6 \%)$ of patients, respectively, after the first series of medication changes were made.

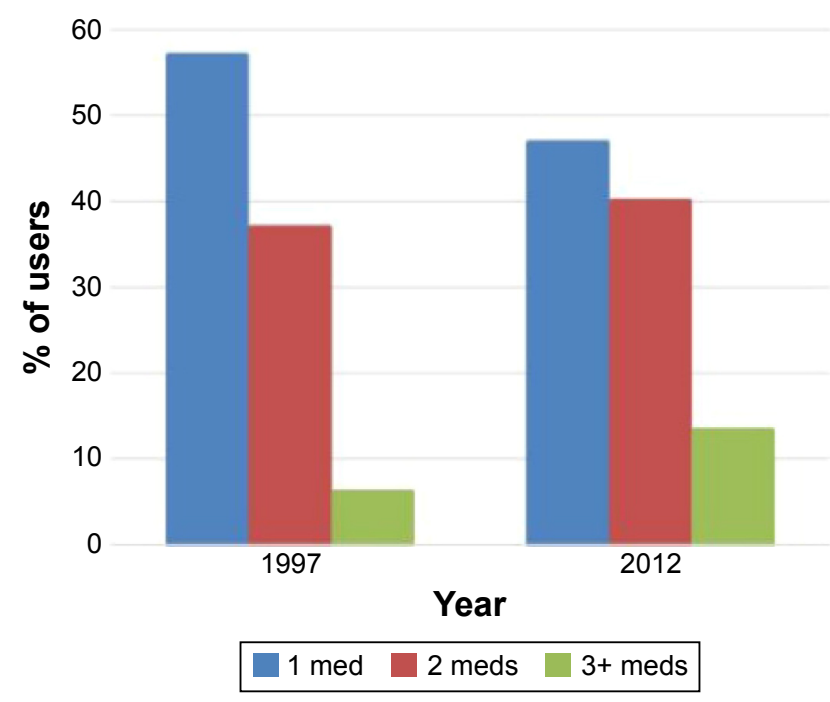

Figure 2 Number of first medications after diagnosis for 1997 vs 2012. Abbreviation: med, medication.
During the overall study period, 5,823 patients were dispensed an ICS. Of these, 3,053 (52.4\%) met 2008 CTS criteria recommended for initiating an ICS. In the analysis to determine those meeting the GOLD guideline criteria for initiating an ICS, although the AECOPD criteria were met by 2,302 patients $(39.5 \%)$, only $47(0.8 \%)$ of all ICS users had been on a LABA or LAMA prior to ICS start. Therefore, $0.3 \%$ of patients dispensed ICS met both required GOLD criteria.

\section{Discussion}

In this study of over 19,000 patients with COPD, of those prescribed a medication after diagnosis, the majority were appropriately started on SABD alone or in combination. Although LABAs or LAMAs alone were uncommonly used as first medications, $28 \%$ of patients were started on an ICS as a first medication. While use of the most basic medications, the SABDs, decreased over the study period, by far the most dramatic increase in use was the combination of LABA and ICS, which rose from 1.2\% for those diagnosed in 1997 to over $27 \%$ for those diagnosed in 2012 (Figure 1). More than half of those started on medications after diagnosis were started on two or more medications. Although the use of single medications as the start of therapy decreased over time, those initiated on three or more medications more than doubled over the study period (Figure 2). After subsequent changes to medication were made, LABA use in the absence of ICS did not increase as expected despite being the next recommended step after basic SABDs according to clinical practice guidelines. ${ }^{22,23}$ Of the changes made to first medications, initiation of an ICS accounted for $83 \%$ of them. Only approximately half of the patients started on ICS during the study period met CTS recommendation criteria, while less than $1 \%$ of the patients met the international GOLD recommendation criteria.

Consistent with current literature, the use of SABD accounted for a significant proportion of medications used in our cohort of new medication users. ${ }^{1,17,24}$ This is expected considering the reasonable relative effectiveness of these classes of medications and their place as first-line therapies in previous and current COPD practice guidelines. ${ }^{22,23}$ It is also expected that LABA and LAMA use would not be as common as first medications, considering their appropriate second-line status when symptoms fail to improve on shortacting agents. However, the use reported in our study for use of these agents without ICS was very low. In a study of US National Ambulatory Medical Care Survey data from 1999 to 2010 , Ford et $\mathrm{al}^{17}$ also evaluated trends in COPD medication 
prescribing visits over time, showing a low and declining proportion of visits for LABA-alone prescriptions and a rise in tiotropium prescription visits from $6.3 \%$ in 2005 to $17.2 \%$ in 2010. The reason for the infrequent use of LABA alone in our study is likely the result of the availability of combination products with ICS and the possible presumption by prescribers that a combination of the two agents is necessarily superior to a LABA alone. This dramatic rise in combination of LABA and ICS prescribing was also seen in the Ford et al ${ }^{17}$ study, which reported an increase in prescription visit rates from $6.2 \%$ in 2001 to $26.7 \%$ in 2010 . Tiotropium was the first LAMA to be marketed in Canada in late 2002. Although the use of tiotropium increased throughout the study period, its use remained low. The practical explanation for this is the fact that tiotropium is not covered by Manitoba's deductiblebased universal drug plan unless the patient has failed to improve on a 3-month trial of ipratropium first. LABAs and LABAs combined with ICS, however, are covered without exception..$^{25}$

In sharp contrast to this, a large proportion of patients $(28 \%)$ were started on an ICS prior to trials of any other medications. As mentioned previously, this was largely driven by a dramatic rise in prescriptions for combination products containing a LABA and ICS. Although not specifically evaluating first medication use, an early study of the Veterans Affairs health system from 1999 to 2003 by Solem et $\mathrm{a}^{18}$ shows some consistency with our findings, reporting that $33 \%$ of patients had received an ICS over a 1-year follow-up in recently diagnosed COPD patients.

Our evaluation of the appropriateness of ICS prescribing showed that approximately half of the patients met Canadian criteria for the initiation of an ICS and only a fraction of a percent met the more stringent criteria recommended by the international GOLD guidelines. A small prospective study in the Canadian provinces of Quebec and Ontario by Bourbeau et $\mathrm{al}^{19}$ reported that nearly two-thirds of COPD patients received a prescription for an ICS despite having only mild COPD that was considered not applicable to guideline-recommended use. Our study of initial medication use provides a large database perspective on a concerning trend of early prescribing of medications with uncertain benefits in early COPD and known harms and cost.

Previous literature on the number of classes of COPD medications prescribed has generally found that in prevalent users, multiple medication use is common. The previously discussed study by Solem et a $1^{18}$ found that $29 \%$ of recently diagnosed COPD patients were prescribed three to four medication classes through the 1-year follow-up of
Veterans Affairs patients. The later study by Ford et $\mathrm{al}^{17}$ based on US National Survey Data from 2010 reported that COPD inhaler prescription visits increased up to three- to fourfold compared to the previous decade, despite overall visits remaining stable. This pattern of multiple inhaler use with an increasing trend over time is not dissimilar to our findings, which analyzed first-time medication prescriptions specifically. Not only did the majority of patients start on multiple medications, but the proportion of patients started on one medication declined over the study period, and the use of three or more initial medications increased significantly over time. The implications of this include effects on adherence, cost, and overall burden of treatment. It is well documented that there is generally poor adherence shown with the use of respiratory medications for COPD and that patients on multiple inhalers or complex regimens are less persistent and adherent than those on single inhalers. ${ }^{17,26}$ A recent Canadian study of COPD medication costs from 2001 to 2010 reported an increase of $\$ 45$ per patient per year of the study.$^{20}$ Considering that the vast majority of those with COPD use multiple classes of chronic non-COPD medications, the addition of multiple inhalers further complicates the broader medication picture, compounding the already large burden of treatment. ${ }^{1,2}$

It is important to note that $31 \%$ of the entire cohort in this study did not have medications started throughout the study period. It was intended that the highly specific inclusion criteria of multiple medical service codes would rule out most patients who were incidentally miscoded for COPD when presenting with nonchronic conditions such as acute bronchitis. It is likely, however, that these scenarios would still occur on occasion and contribute to this nonmedication cohort. It is also possible that patients received samples of COPD medications, but did not have the desire or need to fill future prescriptions. However, although this was an unexpected finding, a Canadian survey of 1,133 patients with a self-reported diagnosis of COPD found that only $75 \%$ of respondents reported taking a medication for COPD. ${ }^{27}$ In addition, a study of US managed care and Medicare patients by Make et $\mathrm{al}^{24}$ reported that more than $60 \%$ of patients received no therapy over a 1-year observation period. It is also of interest that $15.1 \%$ of our cohort that started a medication during the study period stopped their first medication and did not start another. There are several possible explanations for this, including a recognized misdiagnosis of COPD by the prescriber and subsequent discontinuation of therapy, poor adherence to medication by the patient, or a lack of follow-up for medical care despite a diagnosis of COPD. 
To our knowledge, this study is the first to examine the initiation of first medications and the next sequential medication changes for patients with newly diagnosed COPD. A main strength of our study is the longitudinal design of our study using the linked administrative database in a well-defined population over 16 years. Our study has certain limitations that warrant discussion. First, determining medication use based on pharmacy dispensing claims does not allow for assessment of whether the medication was actually taken. However, although actual utilization of medications provides valuable information, it was our intention for this study to focus specifically on prescribing patterns. Similarly, studies of this nature also cannot capture medications that are prescribed but never filled by the patient. This could have the effect of potentially underestimating prescribing estimates and either overestimating or underestimating appropriateness, depending on the medication prescribed. Lastly, the diagnosis of COPD was based on medical billing records, which may have led to possible misclassifications in diagnosis. It is expected, however, that our use of high-specificity case definition criteria will have minimized this. At the same time, it is likely that in this trade-off, some cases of diagnosed COPD will not have been captured.

\section{Conclusion}

This study highlights several important concerns in early COPD medication prescribing patterns. The use of basic firstline medications has declined over time, replaced in large part by combination inhalers that are prescribed inappropriately early without prior trials of other medications with known efficacy. This, combined with the increasing and predominant use of multiple medications at the start of therapy, indicates a significant degree of medication burden in this already complex patient population. Despite the widespread dissemination of both Canadian and GOLD COPD treatment guidelines, clinicians in Manitoba routinely prescribe steroids earlier in the treatment course than recommended. These findings highlight a need for the provision of enhanced education for prescribers to optimize evidence-based management of COPD in the early stages after diagnosis while minimizing the burden of care.

\section{Acknowledgments}

The authors acknowledge the Manitoba Centre for Health Policy for use of data contained in the Population Health Research Data Repository under project (HIPC\# 2015/2016-17). Data used in this study are from the
Population Health Research Data Repository housed at the Manitoba Centre for Health Policy, University of Manitoba, and were derived from data provided by Manitoba Health, Seniors and Active Living.

\section{Disclosure}

The authors report no conflicts of interest in this work.

\section{References}

1. Green ME, Natajaran N, O’Donnell DE, et al. Chronic obstructive pulmonary disease in primary care: an epidemiologic cohort study from the Canadian Primary Care Sentinel Surveillance Network. CMAJ Open. 2015;3(1):E15-E22.

2. García-Olmos L, Alberquilla A, Ayala V, et al. Comorbidity in patients with chronic obstructive pulmonary disease in family practice: a cross sectional study. BMC Fam Pract. 2013;14:11.

3. Penning-van Beest F, van Herk-Sukel M, Gale R, Lammers JW, Herings R. Three-year dispensing patterns with long-acting inhaled drugs in COPD: a database analysis. Resp Med. 2011;105(2):259-265.

4. World Health Organization. The Top 10 Causes of Death. Geneva: World Health Organization; 2014. Available from: www.who.int/ mediacentre/factsheets/fs310/en/. Accessed August 5, 2016.

5. Karner C, Chong J, Poole P. Tiotropium versus placebo for chronic obstructive pulmonary disease. Cochrane Database Syst Rev. 2012;(7): CD009285.

6. Roche N, Chanez P. Bronchodilator combinations for COPD: real hopes or a new Pandora's box? Eur Respir J. 2013;42(6):1441-1445.

7. Falk J. Current issues in managing chronic obstructive pulmonary disease. Pharm Pract Plus. 2015;2(4):26-31.

8. Cheyne L, Irvin-Sellers MJ, White J. Tiotropium versus ipratropium bromide for chronic obstructive pulmonary disease. Cochrane Database Syst Rev. 2013;(9):CD009552.

9. Kew KM, Mavergames C, Walters JA. Long-acting beta2-agonists for chronic obstructive pulmonary disease. Cochrane Database Syst Rev. 2013;(10):CD010177.

10. Farne HA, Cates CJ. Long-acting beta2-agonist in addition to tiotropium versus either tiotropium or long-acting beta2-agonist alone for chronic obstructive pulmonary disease. Cochrane Database Syst Rev. 2015;(10):CD008989.

11. Rodrigo GJ, Plaza V. Efficacy and safety of a fixed-dose combination of indacaterol and glycopyrronium for the treatment of COPD: a systematic review. Chest. 2014;146(2):309-317.

12. Bateman ED, Ferguson GT, Barnes N, et al. Dual bronchodilation with QVA149 versus single bronchodilator therapy: the SHINE study. Eur Respir J. 2013;42(6):1484-1494.

13. Decramer M, Anzueto A, Kerwin E, et al. Efficacy and safety of umeclidinium plus vilanterol versus tiotropium, vilanterol, or umeclidinium monotherapies over 24 weeks in patients with chronic obstructive pulmonary disease: results from two multicentre, blinded, randomised controlled trials. Lancet Respir Med. 2014;2(6):472-486.

14. Calverley P, Anderson JA, Celli B, et al. Salmeterol and fluticasone propionate and survival in chronic obstructive pulmonary disease. N Engl J Med. 2007;356(8):775-789.

15. Kew KM, Seniukovich A. Inhaled steroids and risk of pneumonia for chronic obstructive pulmonary disease. Cochrane Database Syst Rev. 2014;(3):CD010115.

16. Suissa S, Patenaude V, Lapi F, Ernst P. Inhaled corticosteroids in COPD and the risk of serious pneumonia. Thorax. 2013;68(11):1029-1036.

17. Ford ES, Mannino DM, Giles WH, et al. Prescription practices for chronic obstructive pulmonary disease: findings from the national ambulatory medical care survey 1999-2010. COPD. 2014;11(3):247-255.

18. Solem CT, Lee TA, Joo MJ, Lambert BL, Walton SM, Pickard AS. Complexity of medication use in newly diagnosed chronic obstructive pulmonary disease patients. Am J Geriatr Pharmacother. 2012; 10(2):110-122. 
19. Bourbeau J, Sebaldt RJ, Day A, et al. Practice patterns in the management of chronic obstructive pulmonary disease in primary practice: the CAGE study. Can Respir J. 2008;15(1):13-19.

20. Khakban A, Sin DD, Fitzgerald JM, et al. Ten-year trends in direct costs of COPD: a population-based study. Chest. 2015;148(3):640-646.

21. Gershon AS, Wang C, Guan J, Vasilevska-Ristovska J, Cicutto L, To T. Identifying individuals with physician diagnosed COPD in health administrative databases. COPD. 2009;6(5):388-394.

22. O'Donnell DE, Hernandez P, Kaplan A, et al. Canadian Thoracic Society recommendations for management of chronic obstructive pulmonary disease - 2008 update - highlights for primary care. Can Respir J. 2008; 15(Suppl A):1A-8A.

23. Global Initiative for Chronic Obstructive Lung Disease (GOLD), 2015. Global strategy for the diagnosis, management, and prevention of chronic obstructive pulmonary disease [updated 2015]. Available from: http://www.goldcopd.org/. Accessed June 7, 2016.
24. Make B, Dutro MP, Paulose-Ram R, Marton JP, Mapel DW. Undertreatment of COPD: a retrospective analysis of US managed care and medicare patients. Int J Chron Obstruct Pulmon Dis. 2012;7:1-9.

25. Manitoba Drug Benefits \& Interchangeability Formulary. Seniors and Active Living. Winnipeg: Manitoba Health; 2016. Available from: http://www.gov.mb.ca/health/mdbif/. Accessed August 9, 2016.

26. Yu AP, Guerin A, Ponce de Leon D, et al. Therapy persistence and adherence in patients with chronic obstructive pulmonary disease: multiple versus single long-acting maintenance inhalers. J Med Econ. 2011;14(4): 486-496.

27. Public Health Agency of Canada. Fast Facts about Chronic Obstructive Pulmonary Disease (COPD): Data Compiled from the 2011 Survey on Living with Chronic Diseases in Canada. Ottawa: Public Health Agency of Canada; 2013. Available from: www.phac-aspc.gc.ca/ cd-mc/publications/copd-mpoc/ff-rr-2011-eng.php. Accessed August 5, 2016. 


\section{Supplementary material}

Table SI Groupings of COPD medications used in the study analyses

\begin{tabular}{ll}
\hline Grouping by class & Medications \\
\hline SABA & Fenoterol, salbutamol, terbutaline \\
SAMA & Ipratropium \\
Combinations of short-acting agents & Fenoterol/ipratropium, salbutamol/ipratropium \\
Methylxanthines & Aminophylline, oxtriphylline, theophylline \\
LABA & Formoterol, indacaterol, salmeterol \\
LAMA & Glycopyrronium, tiotropium \\
ICS & Beclomethasone, budesonide, ciclesonide, fluticasone, mometasone \\
ICS and LABA combinations & Formoterol/budesonide, formoterol/mometasone, salmeterol/fluticasone
\end{tabular}

Abbreviations: SABA, short-acting $\beta-2$ agonists; SAMA, short-acting muscarinic antagonist; LABA, long-acting $\beta-2$ agonists; LAMA, long-acting muscarinic antagonist; ICS, inhaled corticosteroids.

\section{Publish your work in this journal}

The International Journal of COPD is an international, peer-reviewed journal of therapeutics and pharmacology focusing on concise rapid reporting of clinical studies and reviews in COPD. Special focus is given to the pathophysiological processes underlying the disease, intervention programs, patient focused education, and self management protocols.
Dovepress

This journal is indexed on PubMed Central, MedLine and CAS. The manuscript management system is completely online and includes a very quick and fair peer-review system, which is all easy to use. Visit $\mathrm{http}: / /$ www.dovepress.com/testimonials.php to read real quotes from published authors.

\footnotetext{
Submit your manuscript here: http://www.dovepress.com/international-journal-of-chronic-obstructive-pulmonary-disease-journal
} 